\title{
Effects of aging, phenotype and carbohydrate feeding on caloric efficiency and adiposity in the LA/Ntul//- $c p$ (corpulent) rat
}

\begin{abstract}
Obesity develops in the obese phenotype of the LA/Ntul//-cp (corpulent) specific pathogenfree rat strain by 5 to 6 weeks of age. Groups [n=12 -20 rats/phenotype] of female congenic lean and obese LA/Ntul//-cp (corpulent) rats were fed ad libitum standardized Purina diets for 4,14 , or 24 months or the same diet plus a $16 \%(\mathrm{w} / \mathrm{v})$ sucrose solution supplement from 12 weeks of age, and measures of body weight, caloric intake, and caloric efficiency (CE) determined at each age group. Body weights of lean animals remained similar at all ages studied, while body weights of obese phenotype were significantly greater than their lean littermates at each age studied. The sucrose supplement was without significant effect on final body weights in the lean phenotypes at all ages studied $(\mathrm{p}=$ n.s.) but were associated with greater body weights at ages 4,14 and 24 months of age in the obese phenotype $(\mathrm{p}=<0.05)$. CE was determined as the ratio of $\mathrm{kcal} / \mathrm{gram}$ of body weight per day remained relatively constant in lean animals throughout the age range, but CE was more efficient in the obese phenotype at all ages studied and became progressively more efficient with the sucrose supplement feeding with increasing age. The results of this study indicate that $\mathrm{CE}$ is associated with the predisposition for the development of obesity in the obese phenotype of this strain and likely implicates multiple metabolic factors that contribute to a greater efficiency of energy utilization and or energy conservation in the obese than in the lean phenotype of this strain, and the metabolic impact of added sucrose was associated with an additive impact on the $\mathrm{CE}$ of weight gain and adiposity in the obese phenotype of this congenic rodent strain.
\end{abstract}

Keywords: aging, caloric efficiency, obesity, brown adipose tissue, congenic rodent model
Volume II Issue I - 202I

\author{
Orien L Tulp \\ University of Science Arts and Technology, Montserrat, British \\ West Indies
}

Correspondence: Orien L Tulp, University of Science Arts and Technology, Aspen Business Park, 5400 Ward Road, Building III, Suite 150, Arvada, CO, 80002. USA, Tel 0I-303-352-0II5, Fax 0I-030-37I-0I 17, Email O.tulp@usat.edu

Received: January 13, 2021 | Published: February 09, 2021
Abbreviations: CE, caloric efficiency; BAT, brown adipose tissue; DIT, diet induced thermogenesis

\section{Introduction}

Recent reports indicate that obesity and overweight conditions are rapidly approaching epidemic proportions in Western society and are associated with increased risks for development of cardiovascular and other serious comorbidities. ${ }^{1,2}$ Recent studies in the Wistar rat indicate that diabetogenic diet may predispose to insulin mediated lipid deposition and gender specific regionalization of adiposity in female vs. male animals. ${ }^{3}$ Moreover, the consumption of sugary beverages and its attendant impact on overweight conditions are approaching epidemic proportions in much of Western society. ${ }^{1-3}$ The effects of age and genetic predisposition as independent variables, however, are more difficult to assess in human populations due at least in 1 part to the wide genetic variability present in the general population. The LA/Ntul// $-c p$ (corpulent) rat is a congenic animal model, developed from the LA/N strain at the NIH by Hansen ${ }^{4}$ and Greenhouse ${ }^{5}$ and further characterized by Michaelis ${ }^{6}$ and Tulp. ${ }^{7}$ Obesity occurs in this strain as the result of an autosomal recessive trait, and thus appears in $\sim 25 \%$ of the offspring of heterozygous breeding pairs for the (-cp) trait. ${ }^{4}$ Obese offspring have rarely been observed to reproduce due to endocrinopathies that as yet remain unclear. ${ }^{7}$ The obese phenotype demonstrate hyperinsulinemia, hyper-amylinemia, hyperlipidemia, are normotensive, and are modestly glucose intolerant but remain non-diabetic. ${ }^{6,7}$ The current animals were derived following the $12^{\text {th }}$ backcross at the NIH by Hansen thereby establishing the congenic criterion for the colony and virtually eliminating experimental variability within the strain with the exception of the obese $(-c p)$ characteristic in the obese phenotype.,5 Because the obesity phenotype is expressed as an autosomal recessive trait it occurs with great consistency in $\sim 25 \%$ of the offspring of heterozygous breeders, and thus is an excellent model to investigate the independent variables of age and phenotype on metabolic factors likely to be contributory or causative to the predisposition of obesity and overweight conditions independent of NIDDM. ${ }^{5,6}$ The LA/N background strain was noted for its longevity by Hansen ${ }^{4}$ and in studies by Tulp indicate that this strain ranks among the most long-lived of the obese rodent models yet reported, with some animals surviving beyond 4 years under standard laboratory conditions. ${ }^{7}$ Lean animals have a normal thyroidal response to diet and cold environment, while the dietary and environmental responses in obese animals with the $(-c p)$ trait have been shown to be impaired. $^{7-20}$

Schlafani et al., ${ }^{21,22}$ investigated a broad range of dietary carbohydrate sources and reported that introduction of a $16 \%$ sucrose solution (w/v) as a drinking water supplement can stimulate adaptive thermogenesis in lean rats with only a minimal impact on overall caloric intake. Thus, this approach presents a valuable tool to enable one to assess the impact of modest caloric supplementation on diet induced thermogenesis while exerting minimal effects on body composition in normally lean animals. The impact of moderate sucrose feeding on genetically obese animals and its effects on caloric 
efficiency throughout the life span however have not previously been reported, although a number of studies have contributed to multiple metabolic factors that are likely contributors to the etiology of obesity in this and other models of obesity and overweigh conditions. ${ }^{23-28}$ In other studies, partially hydrolyzed cornstarch was preferred to a significantly greater extent by rats than were sucrose solutions, suggesting that the perceptions or preferences for sweetness in rats may differ from those as perceived by humans. ${ }^{21,22,29}$

Brown adipose tissue [BAT] is a major thermogenic tissue of man and animals, and can be stimulated by $\mathrm{CHO}$ feeding, cafeteria overfeeding, cold exposure and noradrenergic stimulation. ${ }^{29-35}$ In mammalian animals, including humans, BAT normally becomes activated soon after birth and is responsible for heat generation via uncoupling of oxidative phosphorylation, which assists in maintaining body temperature during the early life of the newborn of the species. ${ }^{34}$ BAT depots remain histologically present well into adulthood in many mammalian species studied. Histologically, brown adipocytes differ markedly from white 2 adipocytes on the basis of their smaller size, numerous mitochondria, a centrally located nucleus, and multiple small lipid locules, and sympathetic neuroregulation while in contrast, white adipocytes contain a larger single lipid droplet, a small peripherally located nucleus, few mitochondria, and lack direct neural stimulation. ${ }^{36}$ BAT has been proposed as contributing to an energy balancing mechanism for energy balance especially during caloric overnutrition ${ }^{34}$ while in addition to thyroidal mechanisms that have been demonstrated to respond to both over- and undernutrition by switching from $\mathrm{T} 4$ deiodination from the active form (T3) to the inactive form, reverse T3 (rT3). ${ }^{24}$ The T3 as the active form of the thyroid hormone presumably contributes to a number of metabolic processes including protein turnover, carbohydrate oxidation, and others including the energy wasting hepatic mitochondrial $\alpha$-glycerophosphate dehydrogenase shuttle, which has been shown to correlate with circulating T3 concentrations ${ }^{37}$ and to diminish with aging in activity with advancing age in rats $\mathbf{s}^{37,38}$ and the metabolism of $\alpha$-glycerophosphate was also reported to be decreased in subcutaneous adipose tissue samples obtained from obese subjects. ${ }^{28}$ When brown adipocytes are metabolically active the lipid locules become decreased in size, while in the presence of sympathetic blockade, the lipid locules were of larger cross sectional diameter indicative of decreased thermogenic activity. ${ }^{36}$

In previous studies, early overfeeding has been shown to increase both brown adipocyte number and total interscapular depot mass, and which increased mass may persist well into adulthood of the rat, with the result of a greater potential capacity for noradrenaline and cold stimulated thermogenesis later in life..$^{38}$ IBAT activity is a physiologic component of diet induced thermogenesis [DIT] in man and

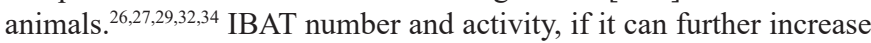
in number and activity later in life in animals and man is unknown. Other biochemical and physiologic mechanisms also likely contribute to buffer energy metabolism, including neuroendocrine, thyroidal, pancreatic, hepatic, and other metabolic processes. ${ }^{17,23-25,27-29,35,37,39,40}$ Historically however, most rodent studies have been conducted in younger male animals, and may not therefore correlate well with the physiologic and neuroendocrine changes that occur in aging and or which may occur in females as a normal consequence of aging at least in part due to gender related endocrinologic differences. The purpose of this study was to assess the effect of aging and obese phenotype on $\mathrm{CE}$ and adiposity and to determine if the impaired responses to diet in the obese phenotype were associated with corresponding changes in $\mathrm{CE}$ which might be correlated with the greater adiposity associated with the $(-c p)$ trait.

\section{Materials and methods}

Groups [ $\mathrm{n}=12-20$ rats/group] of congenic, specific pathogen free (SPF) lean and obese female littermate LA/Ntul//-cp rats were selected from the Drexel University breeding colony at 6 weeks of age and maintained in plastic cages with pine shavings with a reverse light cycle [dark cycle $0800-2000 \mathrm{~h}$ daily] in a temperature-controlled environment $\left[22+/-1{ }^{\circ} \mathrm{C}\right]$ and $40-60 \%$ relative humidity from 6 weeks of age. Animals were fed standard Purina chow \# 5012 and house water ad libitum throughout the study or the same ration plus a $16 \%$ sucrose solution supplement as 3 outlined by Sclafani et al.,, ${ }^{21,22}$ from 12 weeks of age. All housing and laboratory procedures were in accordance with current NIH, AVMA and Institutional Animal care and Use Committee guidelines. Except for the obese trait, biologic variability between offspring of different litters is virtually absent due to the congenic status of this strain and is limited to the potential heterozygosity within the lean littermates of the strain, some of which may carry a homozygous lean trait (--/ --) and some a heterozygous $(-/-c p)$ lean trait. In contrast, all obese littermates are homozygous $(-c p /-c p)$ for the obese characteristic. All breeding pairs consist of heterozygous carriers for the obese trait.

Measures of 24-hour food and fluid intake were obtained by placing individual animals placed in metabolic cages over duplicate 3-day periods, and the data collected for record from the second 3-day period to allow for animals to adequately acclimatize to the metabolic cages. Weights of food consumed were determined on an electronic balance to the nearest 0.1 gram and corrected for spillage. Potential sucrose spillage was corrected by measuring fluid weights minus any spillage with a YSI glucose analyzer fitted with both glucose and sucrose membranes using a glucose oxidase procedure. Food intake is expressed as kcals/rat/day including the actual amounts of sucrose consumed. Computation of caloric efficiency was determined by measuring the kcals of food consumed/gram of body weight per day as reported by Vedula et al. ${ }^{41}$ Measures of animal body weight were obtained with an Ohaus animal balance to the nearest gram at weekly intervals. Statistical analysis was computed with the Minitab Statistics program (Minitab, Inc, State College PA). Descriptive statistics identified outliers within each group and values more than 2 standard deviations from the mean were eliminated from the statistical analysis. Overall variance was assessed by a 1 × 3 ANOVA and the additional interaction of diet on these responses by a 2 × 2 × 3 ANOVA using the General Linear Model, a multiple regression program. Statistical significance was set at alpha of $\mathrm{p}<0.05$ with a Bonferroni correction where $\mathrm{p}=<0.02$.

\section{Results}

In Figure 1, the weights of animals at 4, 14, and 24 months of age are depicted, and indicate that the final body weights of control fed lean animals remained similar throughout the age range, with only a modest trend toward an increase at the oldest age. Addition of the sucrose supplement in lean rats was without effect at the youngest age studied and resulted in only a modest impact trend on weight gain at 14 and 24 months of age. In contrast, obese rats were already significantly heavier regardless of diet by 4 months of age and continued to gain weight at an accelerated rate thereafter becoming more than three times heavier than their lean littermates by 24 months of age ( $\mathrm{p}=<0.01$ at all ages). In the obese phenotype, sucrose supplementation exhibited an even greater impact on weight gain, nearly 3 times as heavy by 14 months of age and 3.25 to 4 times as heavy by 24 months of age. 


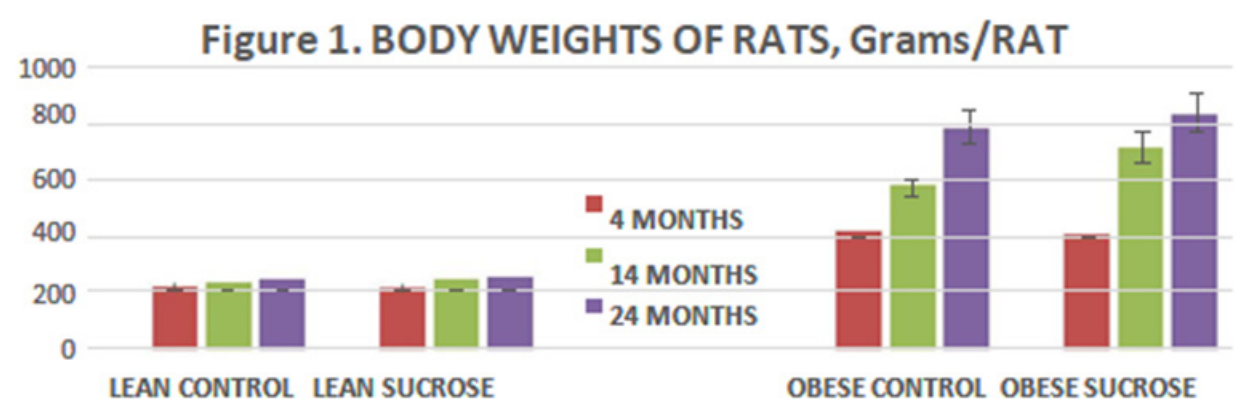

Figure I Body weight of Rats. Data are grams per rat. Lean Control fed rats expressed on the three bars on the left at 4 , I4, and 24 months of age, followed by the next 3 bars fed the 3 fed the sucrose supplement from 12 weeks of age ( $p=$ n.s.); Obese rats are depicted on the right panels similarly fed at 4 , I4 and 24 months of age and fed the sucrose supplement depicted in the far-right bars at 4,14 and 24 months of age $(p=<0.05)$.

Energy intake of animals is depicted in Figure 2 (A) and indicates that energy intake of obese animals was consistently greater in obese than in lean animals throughout the study, weather expressed in absolute terms as kcals/rat/day or relative to body weight as caloric efficiency. In lean animals, the sucrose supplement was without significant effect on total caloric intake or caloric efficiency (Figure 2 (B)) at all ages studied, except for a minor decrease in caloric efficiency in lean sucrose-fed animals at 4 months of age of animals, early in the supplemental feeding regimen. In the obese phenotype, total energy intake was significantly greater than in lean animals at all ages studied. Sucrose supplementation had little if any effect on total energy intake in the obese phenotype. Caloric efficiency (CE) was computed by determining kcal of energy consumed per animal per day divided by the grams body weight of the animal and expressed as a fraction as reported by Vedula et al. ${ }^{41} \mathrm{CE}$ remained relatively stable in lean control animals at all ages studied. CE decreased as reflected by a higher numerical value at the 14 months age in lean animals as expected following introduction of the sucrose supplement and returned toward normal control levels thereafter. In contrast, CE of obese animals was greater, indicated by a lower numerical value initially and continued to increase a reflected by numerically lower values progressively thereafter. Sucrose feeding was suggestive of a modest blip at the onset of the study, but the values tended to deteriorate more rapidly thereafter to less than half the values recorded for lean animals by the end of the study (Figure 2 (B), Right panel $\mathrm{p}=<0.05$ ).

\section{Figure 2A. FOOD INTAKE OF RATS, kcals/rat/day}

140

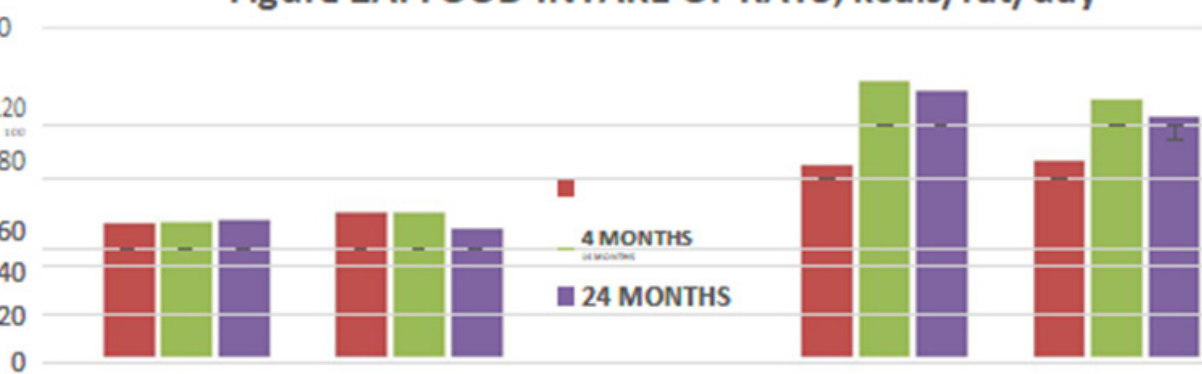

LEAN CONTROL LEAN SUCROSE

OBESE CONTROL OBESE SUCROSE

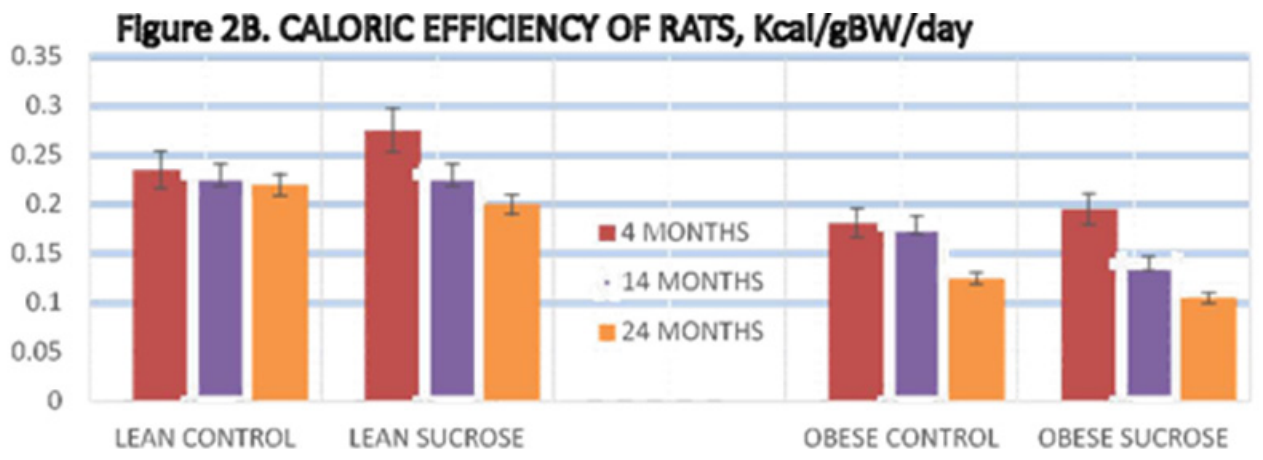

Figure 2 Food Intake of Rats. Data are kcals per rat per day. Lean Control fed rats expressed on the three bars on the left at 4 , I4, and 24 months of age, followed by the next 3 bars fed the 3 fed the sucrose supplement from 12 weeks of age $(p=$ n.s.); Obese rats are depicted on the right panels similarly fed at 4 , 14 and 24 months of age $(p=0.05)$ and fed the sucrose supplement depicted in the far-right bars at 4,14 and 24 months of age ( $p=<0.05)$.

Relative adiposity of rats is depicted in Figure 3 and indicates that the sum of the dorsal and retroperitoneal adipose tissue depots as a percentage of total body weight are generally reflective of the adiposity of the animals of each group and indicate that the relative adiposity of the normally fed lean phenotype tended to increase only modestly and non-significantly from 4 to 24 months of age. Sucrose supplementation exerted only a modest increase in adiposity at each age studied in the lean phenotype during this period of study. In contrast, the obese phenotype demonstrated marked increases in relative adiposity of 10 - fold or greater magnitude at each age studied 
$(\mathrm{p}=<0.01)$ and which increases were somewhat further exaggerated by the sucrose supplementation. In Table I, Measures of 24-hour Urine Glucose remained negative in all rats at all ages, indicating that despite the increased weight gain and adiposity, the obese phenotype remained non-diabetic at 24 months of age. It remains undetermined if the obese animals had remained on the diabetogenic regimen for a longer duration if some animals might have succumbed to development of NIDDM, but based on the adiposity and physiologic changes observed, it remains a possibility. Historically however obese LA/N//-cp animals have not been reported to develop-diet induced NIDDM in our laboratory to date, consistent with other long-term studies with high sucrose content diets in this strain. ${ }^{42}$

\section{Figure 3. RELATIVE ADIPOSITY OF RATS, Grams WAT/g.BWT}

25

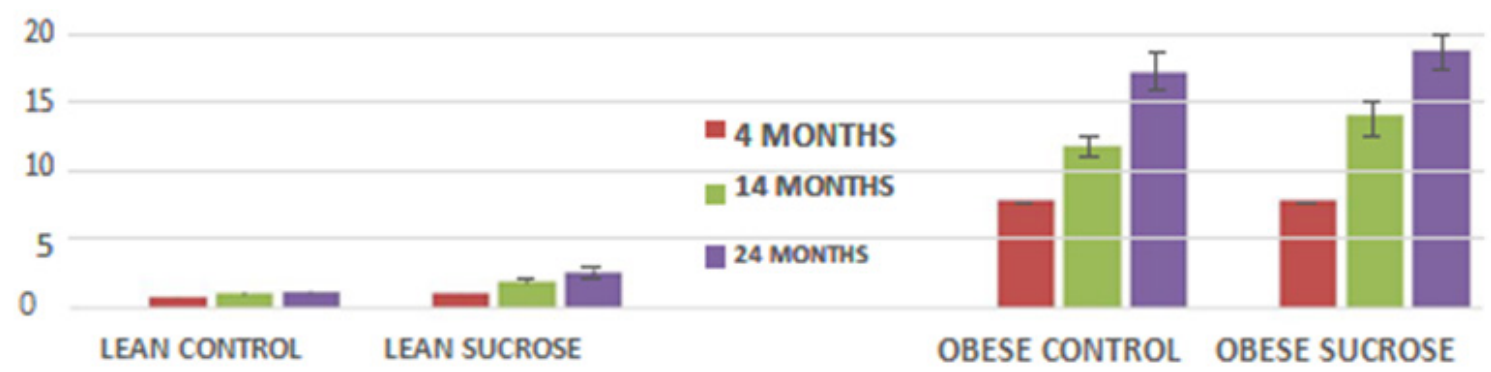

Figure 3 Relative Adiposity of Rats. Data are grams Dorsal plus Retroperitoneal Adipose tissue per gram Body Weight per rat, Lean Control fed rats expressed on the three bars on the left at 4, 14, and 24 months of age, followed by the next 3 bars fed the 3 fed the sucrose supplement from I 2 weeks of age; Lean control rats are similar at all ages ( $p$-n.s.) while in lean sucrose fed rats WAT weight increased at each age $(p=<0.05)$. Obese rats are depicted on the right panels similarly fed at 4, I 4 and 24 months of age and fed the sucrose supplement depicted in the far-right bars at 4, I 4 and 24 months of age and the WAT weights progressively increased at each age studied at each age in both dietary regimens $(p=0.0 \mathrm{I})$.

Table I 24 Hour urine parameters of rats

\begin{tabular}{|c|c|c|c|c|}
\hline \multirow[b]{2}{*}{ Group } & \multirow[b]{2}{*}{$\mathbf{N}$} & \multicolumn{2}{|r|}{ Age } & \multirow[b]{2}{*}{24 Months } \\
\hline & & 4 Months & I 4 Months & \\
\hline & & Glucose & Glucose & Glucose \\
\hline Control Lean & 12 & Negative & Negative & Negative \\
\hline Control Sucrose & 12 & Negative & Negative & Negative \\
\hline Control Obese & 12 & Negative & Negative & Negative \\
\hline Control Sucrose & 12 & Negative & Negative & Negative \\
\hline
\end{tabular}

To determine if Interscapular brown adipose tissue (IBAT) depots reflected a correlation or association with adiposity, the IBAT depots were excised in their entirety and weighed to the nearest $\mathrm{mg}$. on an Ohaus electronic balance. IBAT mass is depicted in Figure 4 (A) and IBAT: Body weight ratio in Figure 4 (B). In Figure 4 (A), IBAT mass of control fed lean animals remained similar at all ages studied and was similar to the IBAT mass of animals of other studies reported by this and other authors. ${ }^{7,8,12,14,15,31-33,35}$ Sucrose supplementation was without a significant effect on IBAT mass at 14 months of age but was associated with significant increases in IBAT mass at 14 and 24 months of age in lean rats when expressed in absolute terms in Figure 4 (A) or relative to body weight in Figure 4 (B). In obese rats, IBAT mass was markedly greater than in lean rats at all ages studied but was not further increased by sucrose feeding thereafter. Not reported here, the hemin pigmentation which gives rise to the brown coloration of the BAT normally observed in IBAT of lean animals was much less intense in the obese than in the lean phenotype due to visibly greater lipid accretion and histologic locularity than normally occurs in IBAT of lean animals. ${ }^{34,36,43}$

Caloric Intake of Rats is depicted in Figure 2 (A) and the Caloric Efficiency (CE) of rats is depicted in Figure 2 (B). The CE of lean rats was similar at all ages studied and was unaffected by the sucrose supplement, indicating that caloric balance and appetite regulation was likely maintained in lean animals. In obese animals, however total caloric intake was significantly greater than in the lean phenotype, consistent with their greater body weight. As in the lean phenotype, the caloric intake of obese sucrose fed animals was similar to the normally fed obese animals. Measures of caloric efficiency are depicted in Figure 2 (B) and indicate that the caloric efficiency of lean animals remained similar at all ages observed and were unaffected by feeding the sucrose-water supplement. This data shows however that the efficiency of energy utilization significantly differed in lean (left panel) vs. obese (right panel) animals. The numerically greater values depicted for the lean animals on the left panel of Figure 2 (B) reflect a less efficient energy utilization, with less weight gained per gram or kcal of energy consumed then occurred in their obese littermates reflected on the right side of Figure 2 (B). In addition, sucrose feeding at $16 \%(\mathrm{w} / \mathrm{v})$ as a fluid supplement was without effect on total caloric intake or caloric efficiency in the lean phenotype. In contrast, caloric intake of obese rats was significantly greater per rat per day that occurred in lean animals, but when expressed as caloric efficiency, energy utilization was significantly more efficient in the obese than in the lean phenotype and became progressively more efficient at each age measured on both dietary regimens. $(p=<0.05)$. Sucrose liquid supplementation at the $16 \%(\mathrm{w} / \mathrm{v})$ resulted in a greater caloric efficiency in the obese phenotype however, consistent with the greater weight gain and adiposity observed in sucrose fed obese animals at both 14 and 24 months of age. Hyperphagia per se was not apparent when expressed relative to body weight in the obese phenotype when consuming the Purina 5012 diet or the Purina 5012 diet plus the $16 \%$ sucrose (w/v) supplement. 


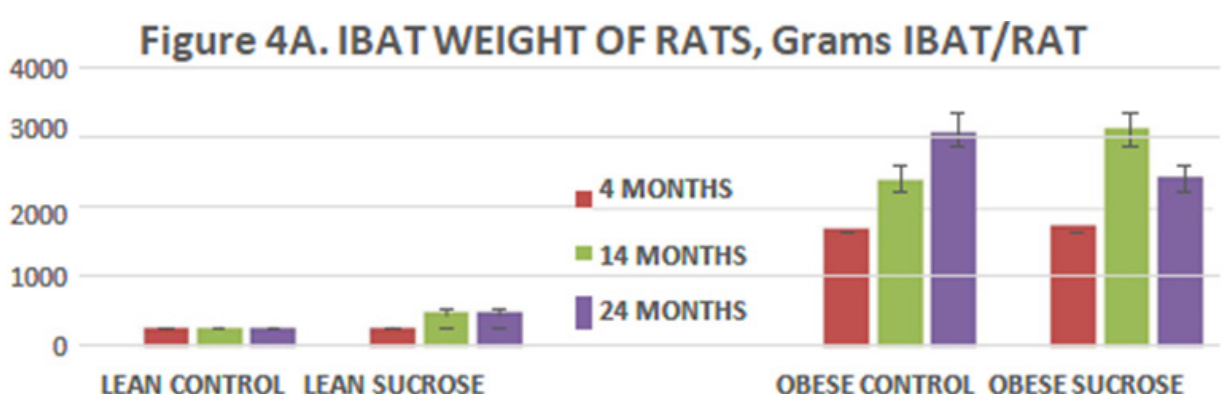

Figure 4 (A) IBAT weights of Rats. Data are grams IBAT per rat, Lean Control fed rats expressed on the three bars on the left at 4 , I4, and 24 months of age and were similar at all age's studies $(p=n$.s.). IBAT weights of lean rats fed the sucrose supplement are depicted in the next panel of 3 bars and indicate that IBAT weights increased at each age studied from 12 weeks of age and were greater at I4 and 24 months of age ( $p=<0.05$ ). IBAT weights of Obese rats are depicted on the right panels similarly fed at 4,14 and 24 months of age and fed the sucrose supplement depicted in the far-right bars at 4 , 14 and 24 months of age and indicate that IBAT weights of obese rats were markedly greater than in lean rats at all ages studied (phenotype effect) and do not appear to have been significantly impacted by the sucrose regimen per se in that IBAT weights were similar in each group at each group regardless of diet. ( $\mathrm{p}=<0.05$ ).

\section{Figure 4B. IBAT WEIGHT OF RATS, Grams IBAT:BW x 10.3}

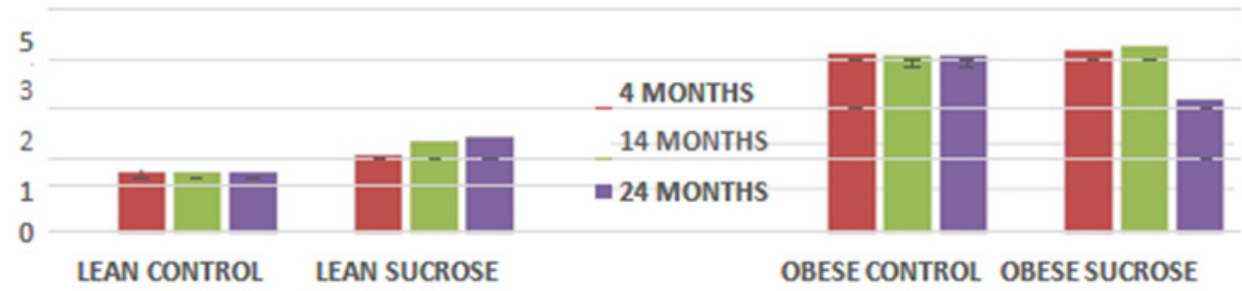

Figure 4 (B) IBAT weights of Rats Relative to Body Weight of Rats. Data are grams IBAT per gram body weight rat $x$ I00, Lean Control fed rats expressed on the three bars on the left at 4,14 , and 24 months of age and are similar at all ages studied ( $p=$ n.s.), followed by the next 3 bars fed the 3 fed the sucrose supplement from 12 weeks of age and indicate that the IBAT increases as a proportion of body weight at each age ( $p=<0.05)$; Obese rats are depicted on the right panels similarly fed at 4, I4 and 24 months of age and fed the sucrose supplement depicted in the far right bars at 4 , I 4 and 24 months of age and indicate that the proportion of IBAT is greater than in lean rats at all ages studied (phenotype effect, $p=<0.05$ ) but remains proportionately similar to body weight at all ages studied.

\section{Discussion}

Obesity occurs in the LA/Ntul//-cp rat as the direct result of an autosomal recessive trait. ${ }^{4,5}$ The results of this study indicate that the obese phenotype of this strain is associated with significant impairments in the efficiency of caloric efficiency. Moreover, this impairment becomes further deranged with aging in this model of obesity. While no biochemical or hormonal measures were reported in this study, in lean Sprague-Dawley rats reported in other studies have shown that thyroidal and neuroendocrine responses contribute to caloric efficiency and are overall those findings were found to be consistent with the findings of this study. ${ }^{23-25,28,32,33,43,44}$

Brown adipose tissue is an active thermogenic tissue in man and animals, and its presence and relative capacity for thermogenic activity may reflect a correlation with the propensity to act as a protective buffer element for adiposity as proposed by Himms-Hagen ${ }^{34}$ and others. ${ }^{30}$ Experimental overnutrition or feeding of protein restricted (protein energy metabolism-inducing) diets in lean weanling (21 day old) Sprague-Dawley rats resulted in marked increases in resting and norepinephrine-stimulated thermogenesis, and in up to 3 -fold increases in interscapular brown adipose number and mass after only 21 days of overfeeding or PEM diets and that the increased IBAT mass, cellularity and thermogenic activity suggesting that its thermogenic activity remained present through adulthood. ${ }^{33,38}$ The increased thermogenic responses to diet and environment occurred in lean but not in genetically obese rats. ${ }^{39}$ Moreover, cold exposure resulted in increases in circulating $\mathrm{T} 3$ in lean but not in obese animals. ${ }^{15}$ In lean animals, exposure to a low protein diet resulted in marked stunting of linear growth, but in obese animals, feeding of the low protein diets were without effect on lean body mass, suggesting that hyperinsulinemia and impaired thyroidal responses characteristic of the obese phenotype suppressed protein turnover thereby consistent with the greater caloric efficiency observed in obese animals and which was likely metabolically associated with a greater magnitude of insulin resistance than occurred in the lean phenotype following introduction of the sucrose supplement. ${ }^{40,43}$

The obese phenotype of the corpulent (-cp) strain has been shown to develop hyperinsulinemia, hyperamylinemia and moderately impaired glucose tolerance, but remain non-diabetic when fed a nutritionally adequate $\operatorname{diet}^{44}$ In the present study, measures of glycemic status were not recorded, but measurements of urine glucose with a YSI glucose analyzer fitted with both glucose and sucrose membranes (to correct for potential sucrose spillage in the urine) remained negative throughout the study, indicative that animals remained non-diabetic at 24 months of age. Compared to lean animals, weight gain in the obese phenotype was disproportionate to energy intake, and the caloric efficiency was markedly improved to nearly twice that of the lean animals when compared to the lean animals on either diet regimen. In other studies, the obesity was also associated with decreased longevity, albeit it considered long for an obese rodent strain. ${ }^{7}$ The longevity of the obese phenotype was also significantly decreased by over $25 \%$ compared to their normally fed lean counterparts, however, with typical longevity of obese phenotype seldom exceeding 36 months while the lean littermates often survived to 48 months or beyond. ${ }^{7}$ The obese of most rodent strains average a 
range of 12 to 15 months, similar to the ages reported for the obese phenotype of the SHR/N-cp strain. ${ }^{6,7}$

The mechanism of the excess weight gain and enhanced caloric efficiency in the obese phenotype is unclear, but it is speculative that it is likely linked at least in part to the hyperinsulinemia and insulin resistance common to the (-cp) trait in this strain. Caloric intake or caloric efficiency was unaffected by aging or sucrose supplementation in the lean phenotype, indicating that appetite regulation per se was not significantly affected in either phenotype. Insulin resistance is known to impact on such diverse processes as glucose oxidation, lipogenesis, sodium-potassium ATPase activity, and protein synthesis and degradation, thereby simultaneously influencing both lipid accretion and protein turnover, the latter being a biochemically expensive process consuming 4 ATPs per peptide bond formed. ${ }^{40,43,44-47}$ Thus, in states of insulin resistance, one might expect the efficiency of energy metabolism to reflect such an economy of energy conservation with a shift toward more efficient protein sparing and resultant greater lipid accretion, especially when associated with impairments in intracellular thyroid hormone metabolism and action. as has been reported above in this rodent strain.

Insulin also impacts on the metabolism and thermogenic activity of brown adipose tissue, both by enhancing lipid locule storage as it does in other lipogenic tissues and organs and by impairing thermogenic activity. Marette et al., ${ }^{11,74,28}$ Bukowieki et al., ${ }^{13}$ and Atgie et al., ${ }^{52}$ reported that there was a major thermogenic defect in brown adipose tissue linked to insulin resistance in the SHR/N-cp rat, which shares the same genetic trait for obesity. ${ }^{4-6}$ Others have shown that peripheral T3 generation from T4 is also impaired in the obese phenotype of the corpulent $(-c p)$ strains following both dietary and cold stimulation, ${ }^{40-48}$ in addition to impaired receptor binding to hepatic binding sites for $\mathrm{T} 3$ and for impaired 5' deiodinase activity, resulting longer T1/2 for hormonal activity in the obese of both cp strains. ${ }^{46-51}$ Thus, the endocrine pathologies evident in the obese phenotype of this strain likely contribute wholly or in part to an enhanced capacity for energy conservation, and for the sucrose enhanced adiposity in the obese phenotype of this strain. Jeyakumar et al., ${ }^{3}$ have reported subtle gender related anthropometric differences in fat deposition in female vs. male Wistar rats fed a high calorie diabetogenic diet that were postulated associated with insulin sensitivity, ${ }^{3}$ and the gender differences in anthropometric fat distributions in humans are also well established.

Central adiposity has often been associated with insulin resistance, where it has been proposed to play roles in promoting lipogenesis and impeding lipolysis in white adipose tissues..$^{1,14,23-26}$ In the present study, weight gain in the obese phenotype was disproportionate to energy intake when compared to that of lean animals that were genetically identical with the exception of the presence of the $-c p$ trait, and the caloric efficiency became further deranged with the addition of the insulinogenic nutrient sucrose, resulting is a still greater magnitude of adiposity and weight gain. It remains unclear if the study had been extended for a longer duration if the sucrose fed obese animals would have eventually developed glycosuria indicative of NIDDM as occurs in the SHR/Ntul//-cp, SKY/N-cp, WKY/N-cp and JCR:LA/N-cp strains which share the same backcrossed (-cp) trait for corpulence, dyslipidemias and numerous common endocrine dysfunctions. ${ }^{5,7,8,12,14,52,53}$ In studies reported by Michaelis however, where LA/N-cp rats were fed isoenergetic high carbohydrate diets containing $54 \%$ carbohydrate as either cooked cornstarch or sucrose for 10.5 months both experimental groups remained euglycemic throughout the study, although diet-induced increases in fasting and glucose stimulated insulin responses and adiposity were reported in sucrose fed animals. ${ }^{39}$
Brown adipose tissue is a major thermogenic tissue in man and animals, where it readily generates heat in the normal state via mitochondrial oxidative dephosphorylation ${ }^{31}$ and its presence when defective secondary to insulin resistance or other defective factors may reflect a correlation along with other metabolic processes with an economy of energy conservation. Regardless of the mechanism, the observations of this study are consistent with the autosomal recessive nature of the $-c p$ trait were associated with a greater economy of caloric efficiency and marked adiposity which was further enhanced by aging and compounded by the addition of a sucrose supplement, but was independent of derangements in appetite dysregulation per se.

\section{Acknowledgments}

The author wishes to acknowledge the contributions of Dr. Susan DeBolt, and Dr. Stephen Dubin, of Drexel University and the late Dr. Otho E Michaelis IV, of the Carbohydrate Nutrition Research Laboratory, Beltsville, MD, USDA for contributions, data collection and management assistance in completion of this research.

\section{Conflicts of interest}

No conflict of interest was reported by the authors.

\section{Funding}

None.

\section{References}

1. Jacks DG, Kerna NA. A Comprehensive Analysis of Obesity Part 1. Overview of Obesity. J Obese Nutr Disord. 2018.

2. Haffner SM. Sex hormones, obesity, fat distribution, type 2 diabetes, and insulin resistance: epidemiological and clinical correlation. Int $J$ Obese. 2000;24:S56-S58.

3. Jeyakumar SM. Diabetogenic diet-induced insulin resistance associates with lipid droplet proteins and adipose tissue secretome, but not with sexual dimorphic adipose tissue fat accumulation in Wistar rats. Biochemistry and Biophysics Reports. 2020;24.

4. Hansen CT. The development of the SHR/N- and LA/N-cp (Corpulent) Congenic Rat Strains. In: New Models of Genetically Obese Rats for Studies in Diabetes, Heart Disease, and Complications of Obesity. NIH publication, Division of Research Services, Veterinary Resources Branch, National Institutes of Health, Bethesda; 1988. p. 7-10.

5. Greenhouse DD, Hansen CT, Michaelis OE IV. Development of Fatty and Corpulent Rat Strains. In: New Models of Genetically Obese Rats for Studies in Diabetes, Heart Disease, and Complications of Obesity. NIH publication, Division of Research Services, Veterinary Resources Branch, National Institutes of Health, Bethesda; 1988. 36 p.

6. Michaelis OE. IV in: New Models of Genetically Obese Rats for Studies in Diabetes, Heart Disease, and Complications of Obesity, Veterinary Resources Branch, Division of Research Services, NIH publication, Bethesda; 1988. p. 13-15.

7. Tulp OL. Characteristics of thermogenesis, obesity, and longevity in the LA/N-cp rat. ILAR News. 1990;32(3):32-39.

8. Tulp OL. Thermogenesis and Obesity in the LA/N-cp and SHR/N -cp Rat Strains", in New Models of Genetically Obese Rats for Studies in Diabetes, Heart Disease, and Complications of Obesity, Veterinary Resources Branch, Division of Research Services, NIH publication, Bethesda; 1988. p. 17-25.

9. Young NL, Tulp OL. The effects of norepinephrine and nutritional status on resting metabolic rates in LA/N-cp rats. Comp Biochem Physiol. 1989;597-603.

10. Tulp OL. Impaired activation of thermogenesis in the corpulent rat. Life Sciences. 1984;35:1699-1705. 
11. Marette A, Tulp OL, Bukowiecki LJ. Mechanism linking insulin resistance to defective thermogenesis in brown adipose tissue of obese diabetic $\mathrm{SHR} / \mathrm{N}$-cp rats. Int J Obese. 1991;15:23-831.

12. Tulp OL, OE Michaelis IV, Servetnick, et al. Nonshivering thermogenesis and obesity in the diabetic SKY/N - cp rat. Comp Biochem Physiol. 1994;107A:195-201.

13. Bukowiecki LJ, Deshaies Y, Collet AJ, et al. Major thermogenic defect associated with insulin resistance in brown adipose tissue of obesediabetic SHR/N -cp rats. Am J Physiol. 1991;261:E204-213.

14. Tulp OL, Stevens C, Barbie OA. Non-shivering thermogenesis and obesity in the adult diabetic Wistar fatty rats. Comp Physiol Pharm. 1989;92A(1):37-41

15. Tulp OL. Metabolic and thermic responses to diet and cold environment $\left(4^{\circ} \mathrm{C}\right)$ in obesity during aging. In: Mini Nutritional Assessment (MNA) in the Elderly: Research and Practice. 173 p.

16. Jones CT, Tulp OL. The effects of controlled exercise on the development of obesity in early obese LA/N-cp (corpulent) rats. Fed Proc. 1983;42(3):535.

17. Tulp OL, DeBolt SP, Einstein GP. Decreased capacity for thermogenesis during aging in lean and obese LA/Ntul//-cp (corpulent) rats. Faseb J. 2021;35(1)

18. Tulp OL, Awan AR, Einstein GP, et al. Enhanced caloric efficiency contributes to adiposity in the LA/N//-cp corpulent rats. Faseb $J$. 2021;35(1).

19. Tulp, OL. The effects of experimental over nutrition on non-shivering thermogenesis and obesity in LA/N-cp rats. Comp Bloch Physiology. 1991;98A:567-574

20. Tulp OL, Shields SJ. Thermogenesis in cafeteria-fed LA/N-cp (corpulent) rats. Nutr Res. 1984;4(2):325-332.

21. Sclafani A, Xenakis S. Sucrose and polysaccharide induced obesity in the rat. Physiol Behav. 1984;32:169-174.

22. Sclafani A. Dietary carbohydrate preferences in the rat. Satellite American Symposium Annual Neuroscience Meeting San Antonio San Antonio TX; 1985.

23. Ravussin EM, Bogardus C, Schwartz RS, et al. Glucose induced thermogenesis and insulin resistance in man. Int J Obesity. 1985;9:103109.

24. Danforth E Jr. The role of thyroid hormones and insulin in the regulation of energy metabolism. Am J Clin Nutr. 1983;38:1006-1017.

25. Jequier E. Energy expenditure in obesity. Clin Endocrinol Metab. $1984 ; 13: 563-580$

26. Marette A, Maurige P, Bukowiecki LJ, et al. Hormonal regulation of brown adipocyte glucose transport in genetic obesity. In: Obesity: Dietary factors and Control, In: DR Romsos, editor. Basel: Karger, 1991. p. 119-130.

27. Galton DA, Bray G. Metabolism of $\alpha$-Glycerol Phosphate in Human Adipose Tissue in Obesity. The Journal of Clinical Endocrinology \& Metabolism. 1967;27(11):1573-1580.

28. Tulp OL, Carlin C. Carbohydrate overnutrition induces increased adiposity in rats. Obesity Update. 1993. p. 1-2.

29. Stock MJ, Rothwell N. Diet Induced thermogenesis and energy flux through brown adipose tissue. In: Proceedings of the XII International Congress of Nutrition, Eds Taylor TG Jenkins NK John Libbey, London; 1985. p. 321-325

30. Tulp OL. The development of brown adipose tissue during experimental overnutrition in rats. Int J Obesity. 1981;5:579-591.
31. Tulp OL, Frink R, Danforth E Jr. Effect of cafeteria-feeding on brown and white adipose tissue cellularity, thermogenesis, and body composition in rats. J Nutr. 1982;112(12): 2250-2260.

32. Tulp OL, Horton ES. The effect of prolonged experimental protein malnutrition and of refeeding on growth and adipose tissue development in the rat. $J$ Nutr. 1981;111(7):1145-1156.

33. Himms-Hagen J. Thermogenesis in BAT as an energy buffer. NEJM. 1983;311:1150-156.

34. Tulp OL, Root D, Frink R. The effect of anti-hypertensive drug treatment on brown adipocyte diameter and locule distribution in rats. Comp Biochem Physiol. 1984;79C(2):317-320.

35. Tatleman HM, Tyzbir RS, Tulp OL. Effects of overfeeding on BAT and liver mitochondrial metabolism and shuttle activity in adult rats. Fed Proc. 1981;40:871.

36. Young RA, Tulp OL, Horton, ES. Thyroid and growth responses of young Zucker obese and lean rats to a low protein-high carbohydrate diet. J Nutr 1980;110:1421-1431.

37. Tulp OL. Decreased protein synthesis and degradation in early obese LA corpulent rats. Fed Proc. 1985;44(4):1501

38. Vedula U, Schnitzer-Polokoff R, Tulp OL. The effect of acarbose on the food intake, weight gain, and adiposity of LA/N-cp rats. Comp Biochem Physiol A Comp Physiol. 1991;100:477-482.

39. Michaelis OE, Ellwood KC, Tulp OL, et al. Effect of feeding sucrose or starch diets on parameters of glucose tolerance in the LA/N-corpulent rat. Nutr Res. 1986;6(2):95-99.

40. Tulp OL. Impaired peripheral thyroid activity, decreased protein turnover, and development of obesity in the LA-corpulent rat. Prog X111 Int Cong Of Nutr. 1985.

41. Tulp OL. Effects of acute starvation on brown adipose tissue status in the rat. Nutr Rept Int. 1983;28(2):227-233.

42. Tulp OL, Brown T. Effect of a Fructan-Chromium Complex on Glycemic Responses of Congenic Obese LA/Ntul/ /-cp Rats. JNHFE. 2016.

43. Tulp OL, Black DE, Outrim D, et al. Peripheral thyroid hormone metabolism and actions (THMA) in lean and obese LA/N -cp rats. FASEB J. 1994.

44. Tulp OL. Sympathetic and Thyroidal Components of Diet-Induced Facultative Thermo genesis in the LA-Corpulent Rat. Brain Res Bull. $1985 ; 348$

45. Tulp OL, Paz Nava M, Black DE, et al. Genetic determinants of T3 receptor affinity and thermogenesis in $\mathrm{LA} / \mathrm{N}-\mathrm{cp}$ rats" Proceedings, XV International Congress of Nutrition Adelaide. International Union of Nutritional Sciences. 1993;1:481.

46. Tulp OL, McKee STD. Triiodothyronine (T3) neogenesis in lean and obese LA/N-cp rats. Bioch Biophys Res Commun 1986;140:134-142.

47. Young RA, Shih-Lieh F, Prosky J, et al. Hepatic Conversion of thyroxine in obese and lean Zucker rats. Am J Physiol. 1987;252:E63-E67,

48. Tulp OL, McKee STD, Michaelis OE IV. Effects of dietary carbohydrate type on T4-5'-deiodinase activity of rats. FASEB J. 1988;2:338.

49. Atgie C, Marette A, Desaultels M, et al. Specific decrease in mitochondrial thermogenic capacity in brown adipose tissue of obese SHR/N-cp rats. Am J Physiol. 1993;2665:1674-1680.

50. Abdoulaye D, Pierce WD, Kelly SE. et al. Abnormal insulin and glucose metabolism in the JCR:LA-corpulent rat. Front Nutr. 2016;3:44. 